\title{
AVALIAÇÃO DA MORBIDADE HOSPITALAR E MORTALIDADE POR NEOPLASIA: 2015 - 2019
}

Evaluation of Hospital Morbity and Neoplasm Mortality: 2015 - 2019

Evaluación de Morbidad Hospitalaria y Mortalidad por Neoplasma: 2015-2019

Tainara Lorena dos Santos Ferreira • Universidade Federal do Rio Grande do Norte-UFRN • Discente da pós-graduação em Saúde Coletiva da UFRN • E-mail: tainara_lorena@hotmail.com

Rita Xavier Barbosa Neta Silva • Discente da pós-graduação em Saúde Coletiva da UFRN・E-mail: ritaxaviersilva29@gmail.com

Thiffany Nayara Bento de Morais • UFRN • Discente da graduação em Enfermagem • E-mail: thiffany.bento.085@ufrn.edu.br

Fábia Barbosa de Andrade • UFRN • Docente da pós-graduação em Saúde Coletiva - E-mail: fabiabarbosabr@gmail.com

Autora correspondente:

Thiffany Nayara Bento de Morais• E-mail: thiffany.bento.085@ufrn.edu.br 


\section{RESUMO}

Introdução: Atualmente, um dos maiores problemas de saúde pública trata-se das doenças crônicas não transmissíveis, que têm gerado elevado número de mortes prematuras. Objetivo: Analisar a morbidade hospitalar e mortalidade por neoplasias na população com faixa-etária entre 10 e 59 anos no Brasil, no período de 2015 a 2019. Metodologia: Trata-se de um estudo quantitativo, do tipo observacional, cujo método de investigação caracteriza-se por um estudo epidemiológico ecológico de série temporal em uma série histórica dos últimos cinco anos (2015-2019) disponíveis em meados de Abril de 2021, e extraídos da base nacional de domínio público do Ministério da Saúde intitulada de Departamento de Informática do Sistema Único de Saúde. Resultados: Observou-se que a distribuição da taxa de mortalidade por neoplasia, por 100.000 habitantes por causa do código relativo à classificação de doenças em população com faixa etária entre 10 e 59 anos, se deu por Neoplasia maligna da mama, como segunda causa de mortes por Neoplasia no quinquênio, seguido por Neoplasia maligna da traqueia, dos brônquios e dos pulmões, Neoplasia maligna do cólon, do reto e do ânus, Neoplasia maligna do estômago e Neoplasia maligna das meninges, do encéfalo e de outras partes do sistema nervoso central. Quanto a taxa de morbidade hospitalar a partir das causas de maior prevalência observa-se que o Leiomioma do útero apresenta maior média no período observado (39.454), mediana de 38.88 e desvio padrão de 2.03, seguido por outras neoplasias in situ e neoplasias benignas e neoplasias de comportamento incerto ou desconhecido com média em 31.724, mediana 30.88 e desvio padrão 0.66. Conclusões: A análise de indicadores da saúde por neoplasias demonstra a tendencia crescente no quinquênio da morbidade hospitalar e mortalidade. De modo que se destacam que sejam alvo de maiores pesquisas e atenções.

Palavras-Chave: Mortalidade; Morbidade; Neoplasia; Avaliação em Saúde.

\section{ABSTRACT}

Introduction: Currently, one of the biggest public health problems is chronic noncommunicable diseases, which have generated a high number of premature deaths. Objective: To analyze hospital morbidity and mortality by neoplasms in the population aged between 10 and 59 years in Brazil, in the period from 2015 to 2019 . Methodology: This is a quantitative, observational study, whose research method is characterized by an ecological epidemiological study of time series in a historical series of the last five years (2015-2019) available in mid-April 2021, and extracted from the national public domain base of the Ministry of Health entitled Department of Informatics of the Unified Health System. Results: It was observed that the distribution of the mortality rate due to neoplasia, per 100,000 inhabitants due to disease classification code in a population aged between 10 and 59 years, was due to malignant neoplasm of the breast, as the second leading cause of deaths due to eoplasia in the five-year period, followed by malignant neoplasm of the trachea, onchi and lungs, malignant neoplasm of the colon, rectum and anus, malignant oplasm of the stomach and malignant neoplasm of the meninges, brain and other ts of the central nervous system. Regarding the hospital morbidity rate from the t prevalent causes, it is observed that the uterus leiomyoma has the highest 
average in the period observed $(39,454)$, median of 38.88 and standard deviation of 2.03 , followed by other in situ neoplasms and benign neoplasms and neoplasms of uncertain or unknown behavior with a mean of 31,724, a median of 30.88 and a standard deviation of 0.66. Conclusions: The analysis of health indicators for neoplasms shows the growing trend in the five years of hospital morbidity and mortality. So that they stand out that they are the target of more research and attention.

Keywords: Mortality; Morbidity; Neoplasia; Health Evaluation.

\section{RESUMEN}

Introducción: Actualmente, uno de los mayores problemas de salud pública son las enfermedades crónicas no transmisibles, las cuales han generado un elevado número de muertes prematuras. Objetivo: Analizar la morbilidad y mortalidad hospitalaria por neoplasias en la población de 10 a 59 años en Brasil, en el período de 2015 a 2019. Metodología: Se trata de un estudio observacional cuantitativo, cuyo método de investigación se caracteriza por un estudio epidemiológico ecológico de series de tiempo en una serie histórica de los últimos cinco años (2015-2019) disponible a mediados de abril de 2021, y extraída de la base de dominio público nacional. del Ministerio de Salud titulado Departamento de Informática del Sistema Único de Salud. Resultados: Se observó que la distribución de la tasa de mortalidad por neoplasia, por 100.000 habitantes por código de clasificación de enfermedades en una población de entre 10 y 59 años, se debió a neoplasia maligna de mama, como segunda causa de muerte. debido a neoplasia en el período de cinco años, seguida de neoplasia maligna de la tráquea, bronquios y pulmones, neoplasia maligna del colon, recto y ano, neoplasia maligna del estómago y neoplasia maligna de las meninges, cerebro y otras partes del sistema nervioso central. En cuanto a la tasa de morbilidad hospitalaria por las causas más prevalentes, se observa que el leiomioma de útero tiene el promedio más alto en el período observado (39.454), mediana de 38,88 y desviación estándar de 2,03, seguido de otras neoplasias in situ y neoplasias y neoplasias benignas de comportamiento incierto o desconocido con una media de 31.724, una mediana de 30,88 y una desviación estándar de 0,66. Conclusiones: El análisis de los indicadores de salud por neoplasias muestra la tendencia creciente en los cinco años de morbilidad y mortalidad hospitalaria. Para que destaquen que son objeto de más investigación y atención.

Palabras clave: Mortalidad; Morbosidad; Neoplasia; Evaluación de salud. 


\section{Introdução}

Atualmente, um dos maiores problemas de saúde pública trata-se das doenças crônicas não transmissíveis, que têm gerado elevado número de mortes prematuras, perda de qualidade de vida (com alto grau de limitação e incapacidade para as atividades de vida diária), além de impactos econômicos para famílias, comunidades e a sociedade em geral1,2. A Organização Mundial da Saúde (OMS) inclui como Doenças Crônicas do Aparelho Circulatório (DCNT) as doenças do aparelho circulatório (cerebrovasculares, cardiovasculares), neoplasias, doenças respiratórias crônicas e diabetes mellitus ${ }^{2,3}$.

A OMS estima ainda que no mundo haverá, em 2030, 27 milhões de casos incidentes de todos os tipos de câncer, 17 milhões de mortes e 75 milhões de pessoas vivendo anualmente com essa doença ${ }^{4}$, que caracteriza-se como uma doença causada por meio da divisão desordenada das células devido a falhas no sistema de regulação do ciclo celular e pela capacidade de invadir outras estruturas orgânicas, desencadeando a formação de tumores com características de malignidade ${ }^{5}$.

O câncer é uma doença multicausal e sua relação com fatores de risco ambientais, culturais, socioeconômicos, estilos de vida (principalmente obesidade, tabagismo, consumo de álcool, inatividade física e dieta não saudável), além dos fatores genéticos e o envelhecimento populacional, é conhecida ${ }^{3-5}$. Globalmente, as transições demográficas e epidemiológicas sinalizam para a importância crescente do câncer nas próximas décadas ${ }^{6-9}$.

Sabe-se que as neoplasias apresentam altas taxas de mortalidade no Brasil e elevados custos clínicos assistenciais, todavia, a magnitude dos agravos varia de acordo com a localização primária do tumor, o gênero do paciente e a região do país. Cabe destacar ainda que diversos fatores de risco e de prevenção para as neoplasias, e também de métodos terapêuticos, já estão estabelecidos e apresenta-se como fundamental a aplicação de maneira universal e equânime com vistas ao controle deste ravo $^{10}$. A mortalidade por doenças crônico-degenerativas vem mostrando uma censão progressiva no Brasil, destacando-se as neoplasias malignas entre as cipais causas de morte. 
As diferenças geográficas dessas taxas podem estar relacionadas às características socioeconômicas. Para incidência, há associação com as prevalências do consumo de alimentos não saudáveis, obesidade, tabagismo, entre outros ${ }^{11}$. Para mortalidade, parece decorrer da desigualdade no acesso aos serviços de saúde, dificultando diagnóstico precoce e tratamento oportuno ${ }^{12}$. Uma vez que no Brasil há evidências dessas disparidades entre os estados ${ }^{13}$, o objetivo foi analisar a morbidade hospitalar e mortalidade por neoplasias na população com faixa-etária entre 10 e 59 anos no Brasil, no período de 2015 a 2019.

\section{Metodologia}

Trata-se de um estudo quantitativo, do tipo observacional, cujo método de investigação caracteriza-se por um estudo epidemiológico ecológico de série temporal, com uso de dados de base populacional dos sistemas de informação: Sistema de Informações Hospitalares do Sistema Único de Saúde (SIH/SUS) e Sistema de Informação sobre Mortalidade (SIM) em uma série histórica dos últimos cinco anos (2015-2019) disponíveis em meados de Abril de 2021, e extraídos da base nacional de domínio público do Ministério da Saúde intitulada DATASUS - Departamento de Informática do SUS.

Destarte que esta pesquisa se deu a partir de dados disponíveis em âmbito nacional; para tanto, optou-se por utilizar como unidades de análise o território nacional e as unidades da federação, optando-se como população a faixa etária com idade entre dez (10) e cinquenta e nove (59) anos, agrupando para fins de análise a cada dez anos.

Como objetos deste estudo foram observados os indicadores de saúde: Taxa de Mortalidade e taxa de morbidade hospitalar por neoplasias (Capítulo II do Classificação Internacional de Doenças - CID-10) em população com faixa etária entre 10 e 59 anos de idade por Unidade da Federação do Brasil, extraídos do DATASUS través do SIM e SIH-SUS, respectivamente, referentes ao período histórico dos anos 2015 a 2019. Para cálculo de tais indicadores utilizou-se como denominador a pulação de mesma faixa etária pelo censo de 2010, igualmente extraído pelo TASUS e utilizou-se como base populacional 100.000 habitantes. 
Concernente às causas da Mortalidade pelo Capítulo II - Neoplasias, optou-se por avaliar as cinco principais causas através da seleção no DATASUS por CAUSA CID-BR-10, de modo que se obteve como principal causa: Restante de neoplasias malignas (052) - descrita a partir dos códigos do CID-10 (C17, C23-C24, C26-C31, C37-C41, C44C49, C51-C52, C57-C60, C62-C66, C68-C69, C73-C81, C88-C89, C96-C97), que em alguns versam sobre localizações mal/não definidas/especificadas. Deste modo, se definiu avaliar as 5 CAUSAS CID-BR-10 seguintes, mantendo assim: Neoplasia maligna da mama, Neoplasia maligna da traquéia, dos brônquios e dos pulmões, Neoplasia maligna do cólon, do reto e do ânus, Neoplasia maligna do estômago e Neoplasia maligna das meninges, do encéfalo e de outras partes do sistema nervoso central.

Quanto a Morbidade hospitalar pelo Capítulo II - Neoplasias, optou-se por avaliar as cinco principais causas através da seleção no DATASUS por internações, de modo que se obteve como principais causas: Leiomioma do útero, outras neoplasias in situ e neoplasias benignas e neoplasias de comportamento incerto ou desconhecido, neoplasia maligna de mama, Neoplasias malignas de outras localizações, de localização mal definida, secundárias e de localização não especificada e Neoplasia maligna de cólon.

Para tratamento e análise dos dados, seguiu-se que após coleta de dados dos Sistemas de Informação acima descritos, cada arquivo extraído recebeu tratamento no Microsoft Excel, sendo ainda calculadas os indicadores de saúde acima descritos. As tabelas, os gráficos foram gerados pelo programa Microsoft Excel 2013. Foram calculadas estatísticas descritivas (média e desvio padrão, mediana e intervalo interquartil, mínimos e máximos, para as variáveis quantitativas; e frequências, para as variáveis qualitativas) através do software Statistical Package for the Social Sciences (SPSS).

No que concerne aos aspectos éticos e legais desta pesquisa, a resolução $\mathrm{n}^{\circ} 510$, de 07 de abril de $2016^{14}$, versa que pesquisas que utilizem informações de domínio público e pesquisas com bancos de dados, que possuem informações agregadas e não há identificação individual, não necessitam de registro e avaliação pelo sistema Comitê e Ética em Pesquisa / Comissão Nacional de Ética em Pesquisa. 


\section{Resultados}

Sabe-se que a avaliação de indicadores de saúde tem ganhado relevância no cenário atual uma vez que permite o monitoramento de condições de saúde da população e de ações programáticas. Neste ínterim, relacionado ao capítulo II do CID10 que versa sobre neoplasias, esta pesquisa traz a avaliação a partir dos indicadores de morbidade hospitalar e mortalidade do último quinquênio disponível no SIM.

Nesta perspectiva, a Figura 1 traz a taxa de mortalidade por neoplasias no período de 2015-2019, apresentando Neoplasias com taxa de mortalidade de 172,81 para 100.000 habitantes na série histórica observada. Para além disso destaca-se entre as principais causas de mortes observadas, a codificação de 052 - Restante de neoplasias malignas (052) - descrita a partir de códigos do CID-10 que em alguns versam sobre localizações mal/não definidas/especificadas.

Observou-se ainda Neoplasia maligna da mama, como segunda causa de mortes por neoplasia no quinquênio, seguido por Neoplasia maligna da traqueia, dos brônquios e dos pulmões, Neoplasia maligna do cólon, do reto e do ânus, Neoplasia maligna do estômago e Neoplasia maligna das meninges, do encéfalo e de outras partes do sistema nervoso central $(10,39)$.

Figura 1. Distribuição da taxa de mortalidade por neoplasia, por 100.000 habitantes por Causa CID-BR-10 em população com faixa etária entre 10 e 59 anos. Brasil, 2015-2019. Natal-RN 2021.

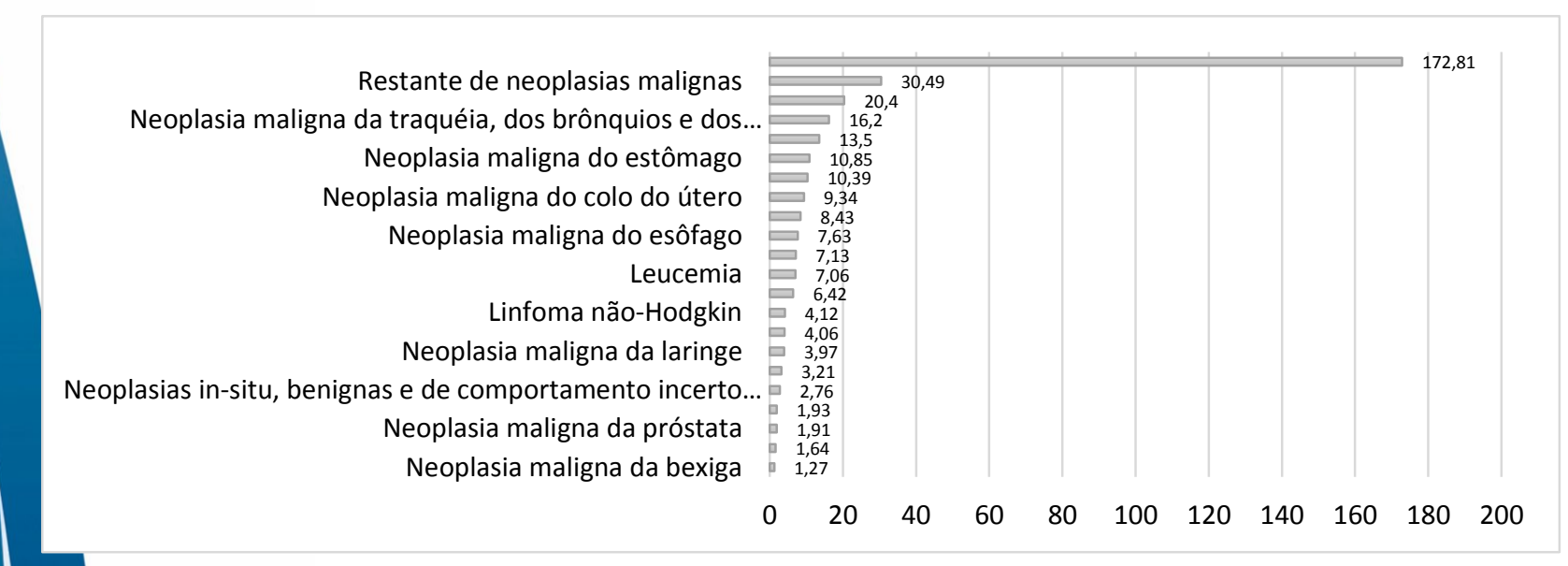

Fonte: Sistema de Informações sobre Mortalidade - SIM/DATASUS. 
Tabela 1. Taxa de mortalidade por 100.000 habitantes das cinco causas mais prevalentes nas unidades da federação do Brasil no período de 2015 a 2019. Natal-RN, 2021.

\begin{tabular}{|c|c|c|c|c|c|}
\hline $\begin{array}{l}\text { UNIDADES DA } \\
\text { FEDERAÇÃO }\end{array}$ & $\begin{array}{l}\text { NEOPLASIA } \\
\text { MALIGNA } \\
\text { DA MAMA }\end{array}$ & $\begin{array}{c}\text { NEOPLASIA MALIGNA } \\
\text { DA TRAQUÉIA, } \\
\text { BRONQUIOS E } \\
\text { PULMÕES }\end{array}$ & $\begin{array}{l}\text { NEOPLASIA } \\
\text { MALIGNA DO } \\
\text { CÓLON, RETO } \\
\text { E ÂNUS }\end{array}$ & $\begin{array}{l}\text { NEOPLASIA } \\
\text { MALIGNA } \\
\text { DO } \\
\text { ESTÔMAGO }\end{array}$ & $\begin{array}{c}\text { NEOPLASIA } \\
\text { MALIGNA } \\
\text { MENING, ENCÉF } \\
\text { E OUT PARTES } \\
\text { SNC }\end{array}$ \\
\hline Rondônia & 15.48 & 11.32 & 8.64 & 9.85 & 9.53 \\
\hline Acre & 13.90 & 15.26 & 5.31 & 14.31 & 7.49 \\
\hline Amazonas & 14.83 & 9.81 & 8.09 & 15.84 & 7.29 \\
\hline Roraima & 14.20 & 11.32 & 9.54 & 11.09 & 8.43 \\
\hline Pará & 11.76 & 8.75 & 6.51 & 12.66 & 7.59 \\
\hline Amapá & 12.24 & 9.26 & 5.22 & 17.62 & 8.96 \\
\hline Tocantins & 14.52 & 11.20 & 8.45 & 8.52 & 10.04 \\
\hline Maranhão & 10.25 & 8.12 & 5.17 & 8.00 & 6.12 \\
\hline Piauí & 16.61 & 10.83 & 6.60 & 7.69 & 9.62 \\
\hline Ceará & 19.34 & 14.44 & 8.35 & 12.38 & 9.55 \\
\hline $\begin{array}{l}\text { Rio Grande do } \\
\text { Norte }\end{array}$ & 19.16 & 15.18 & 9.62 & 11.77 & 8.71 \\
\hline Paraíba & 14.92 & 13.14 & 7.64 & 10.64 & 11.36 \\
\hline Pernambuco & 20.20 & 13.17 & 9.85 & 10.70 & 9.71 \\
\hline Alagoas & 14.96 & 12.14 & 5.83 & 6.69 & 9.13 \\
\hline Sergipe & 14.70 & 8.17 & 6.40 & 5.22 & 5.54 \\
\hline Bahia & 17.35 & 9.84 & 8.53 & 9.20 & 8.76 \\
\hline Minas Gerais & 18.64 & 13.96 & 13.25 & 9.25 & 9.95 \\
\hline Espírito Santo & 22.73 & 14.90 & 14.33 & 13.17 & 10.58 \\
\hline Rio de Janeiro & 30.05 & 18.68 & 18.14 & 10.81 & 10.36 \\
\hline São Paulo & 22.68 & 18.27 & 18.13 & 11.47 & 11.00 \\
\hline Paraná & 21.62 & 19.10 & 16.69 & 12.91 & 13.92 \\
\hline Santa Catarina & 23.04 & 26.80 & 16.21 & 14.06 & 12.93 \\
\hline Rio Grande do Sul & 23.37 & 33.02 & 19.16 & 10.41 & 13.69 \\
\hline $\begin{array}{l}\text { Mato Grosso do } \\
\text { Sul }\end{array}$ & 19.84 & 18.08 & 15.18 & 10.08 & 11.14 \\
\hline Mato Grosso & 17.52 & 14.13 & 11.89 & 9.78 & 9.35 \\
\hline Goiás & 21.41 & 16.42 & 14.64 & 9.44 & 11.42 \\
\hline Distrito Federal & 21.94 & 9.88 & 16.41 & 10.58 & 12.10 \\
\hline BRASIL & 20.40 & 16.20 & 13.50 & 10.85 & 10.39 \\
\hline
\end{tabular}

Fonte: Sistema de Informações sobre Mortalidade - SIM/DATASUS.

Relacionado a distribuição da taxa de mortalidade por unidades da federação, neoplasia maligna da mama, destaca-se as unidades da federação da região Sudeste e Sul apresentando resultados de indicadores maiores que nível nacional, a citar, Rio de Janeiro (30.05), Rio Grande do Sul (23.27) e Santa Catarina (23.04). Quanto a neoplasia maligna da traqueia, dos brônquios e dos pulmões, as unidades da federação que apresentam as maiores mortalidades concentram-se na região Sul, Rio Grande do Sul (33.02), Santa Catarina (26.80) e Paraná (19.10). Relacionado a neoplasia maligna do cólon, reto e do ânus, Rio Grande do Sul (19.16), Rio de Janeiro (18.14) e São Paulo (18.13) presentam-se como unidades da federação com maiores taxas de mortalidade. ncernente a neoplasia maligna do estômago os maiores resultados concentram-se da ião Norte, Amapá (17.62), Amazonas (15.84), Acre (14.31). E, quanto a neoplasia ligna das meninges, do encéfalo e de outras partes do sistema nervoso central as maiores 
taxas concentram-se na região Sul - Paraná (13.92), Rio Grande do Sul (13.69) e Santa Catarina $(12,93)$.

Figura 2. Distribuição da taxa de mortalidade por neoplasia por Causa CID-BR-10 em população com faixa etária entre 10 e 59 anos. Brasil, 2015-2019. Natal-RN, 2021.

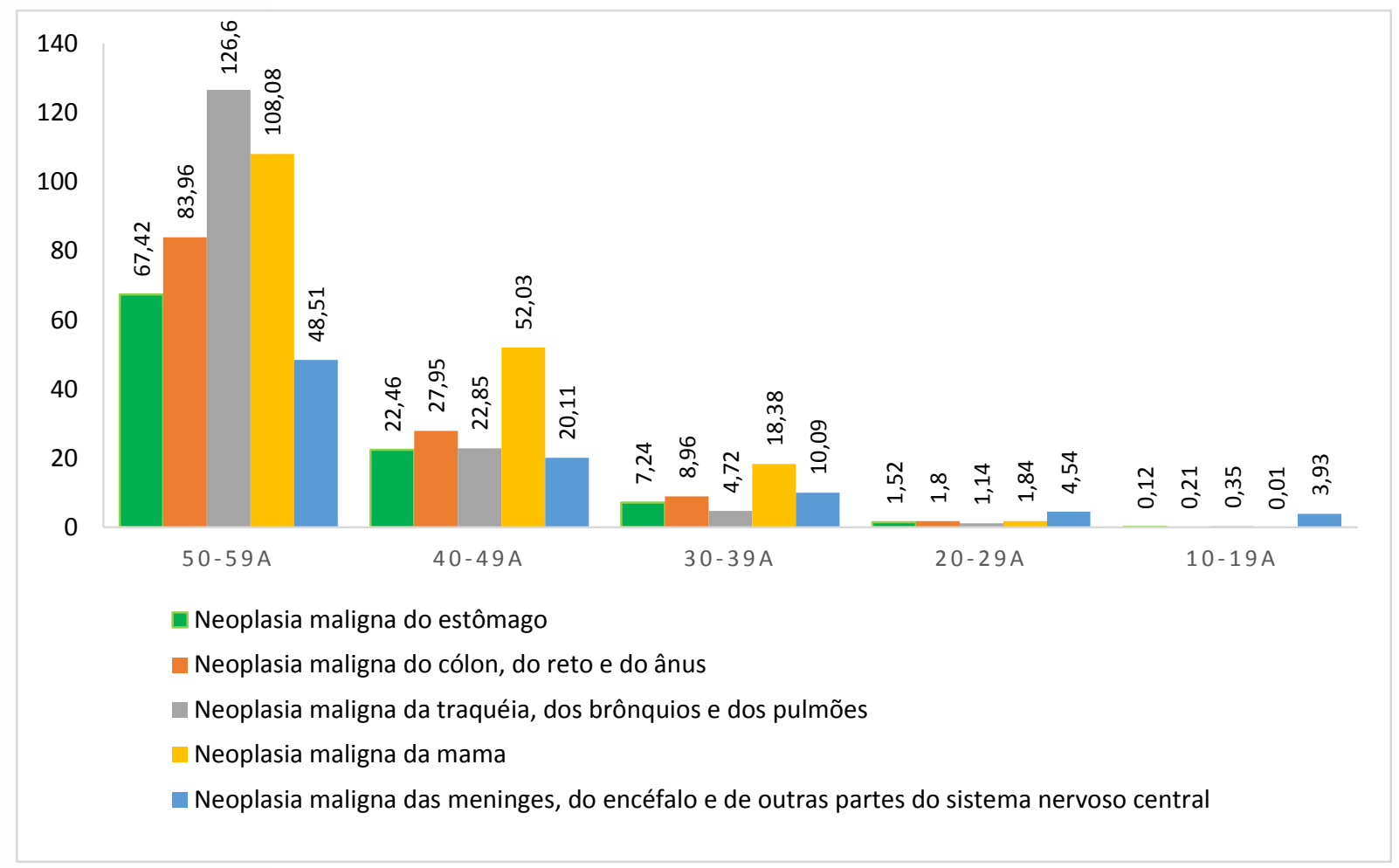

Fonte: Sistema de Informações sobre Mortalidade - SIM/DATASUS.

Relacionado a taxa de mortalidade por neoplasias pela população no período observado, Figura 2, verificou-se o aumento das taxas de mortalidade nos grupos etários de maior faixa etária, todavia, as principais causas de mortalidade variam significativamente.

$\mathrm{Na}$ população com faixa etária entre 50 e 59 anos observa-se maior taxa de mortalidade relacionada a neoplasia maligna da traqueia, dos brônquios e dos pulmões, seguido por neoplasia maligna de mama e neoplasia maligna do cólon, do reto e do ânus. Para a faixa etária entre 40 e 49 anos observa-se maior taxa de mortalidade relacionada neoplasia maligna de mama, seguida por neoplasia maligna cólon, do reto e do ânus e neoplasia maligna da traqueia, dos brônquios e dos Imões. Quanto a faixa etária entre 30 e 39 anos observa-se maior taxa de mortalidade neoplasia maligna de mama, seguida por neoplasia maligna das meninges, do 
encéfalo e de outras partes do sistema nervoso central, e neoplasia maligna do cólon, do reto e do ânus. Para a faixa etária entre 20 e 29 anos e faixa etária entre 10 e 19 anos destaca-se a neoplasia maligna das meninges, do encéfalo e de outras partes do sistema nervoso central.

Quanto à morbidade hospitalar sobre o capítulo II do CID-10 pela lista de morbidade, Figura 3, observa-se Leiomioma do útero apresentando maior taxa de morbidade hospitalar por 100.000 habitantes no período observado (197.28), seguido por outras neoplasias in situ e neoplasias benignas e neoplasias de comportamento incerto ou desconhecido (158.63), neoplasia maligna de mama (108.45), Neoplasias malignas de outras localizações, de localização mal definida, secundárias e de localização não especificada (67.59) e Neoplasia maligna de cólon (56.41).

Figura 3. Distribuição da taxa de morbidade hospitalar por neoplasia, por 100.000 habitantes por Causa CID-BR-10 em população com faixa etária entre 10 e 59 anos. Brasil, 2015-2019. Natal-RN, 2021.

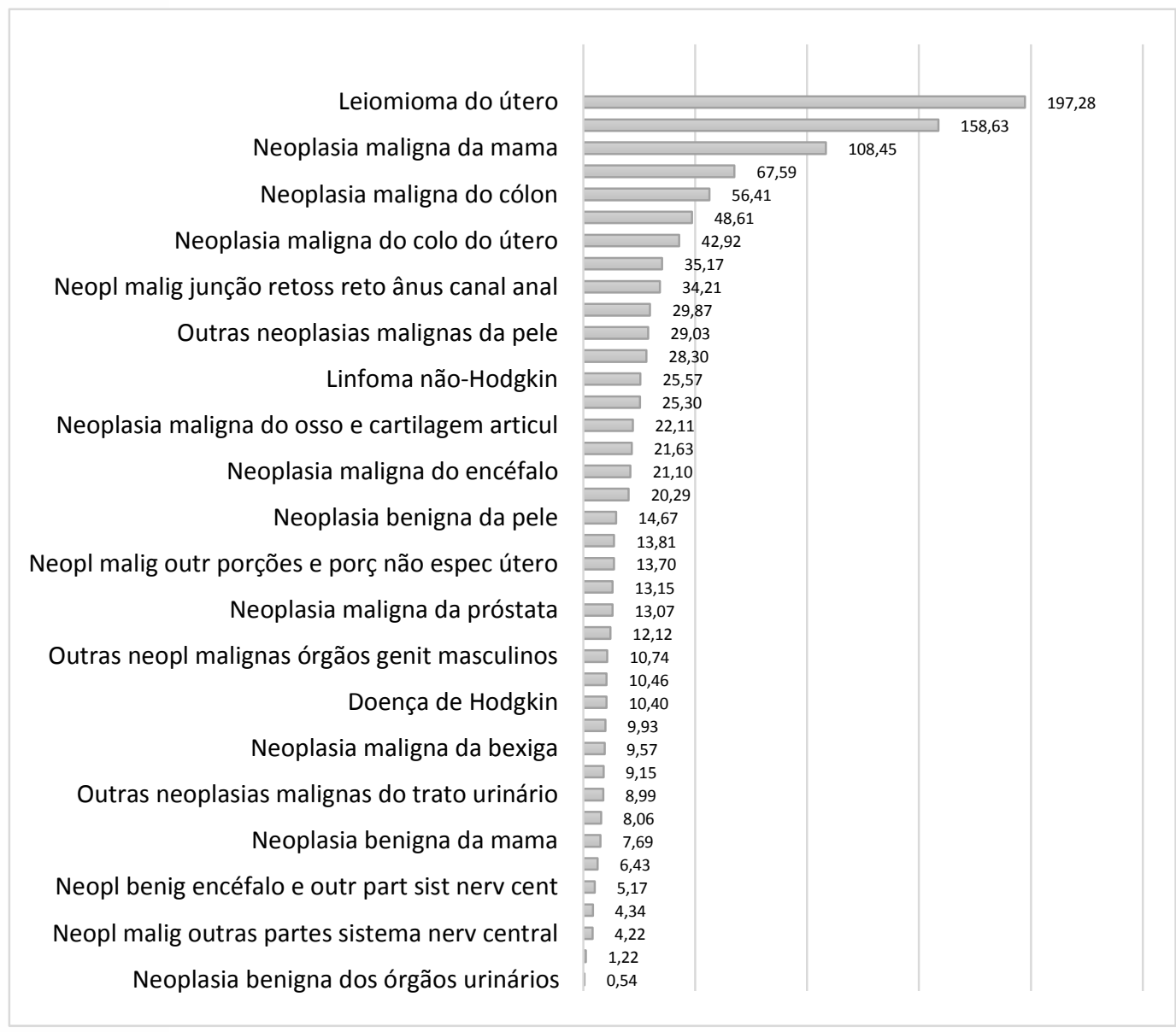

Fonte: Sistema de Informações sobre Morbidade - SIM/DATASUS. 
Tabela 2 - Distribuição do Taxa de Morbidade Hospitalar segundo as causas de maior prevalência, 2015 - 2019, Brasil, 2021. Natal-RN 2021.

\begin{tabular}{|c|c|c|c|}
\hline LISTA MORB CID-10 & MÉDIA & $\begin{array}{l}\text { DESVIO } \\
\text { PADRÃO }\end{array}$ & MEDIANA \\
\hline Neoplasia maligna do cólon & 11.282 & 0.53 & 11.21 \\
\hline Neoplasia maligna da mama & 21.69 & 1.02 & 21.69 \\
\hline $\begin{array}{l}\text { Neoplasias malignas de outras localizações, de } \\
\text { localização mal definida, secundárias e de } \\
\text { localização não especificada }\end{array}$ & 13.518 & 0.43 & 13.43 \\
\hline Leiomioma do útero & 39.454 & 2.03 & 38.88 \\
\hline $\begin{array}{l}\text { Outras neoplasias in situ e neoplasias benignas e } \\
\text { neoplasias de comportamento incerto ou } \\
\text { desconhecido }\end{array}$ & 31.724 & 0.66 & 30.88 \\
\hline
\end{tabular}

Fonte: Sistema de Informações sobre Morbidade - SIM/DATASUS.

Quanto a taxa de morbidade hospitalar a partir das causas de maior prevalência observa-se que o Leiomioma do útero apresenta maior média no período observado (39.454), mediana de 38.88 e desvio padrão de 2.03, seguido por outras neoplasias in situ e neoplasias benignas e neoplasias de comportamento incerto ou desconhecido com média em 31.724, mediana 30.88 e desvio padrão 0.66, neoplasia maligna da mama, neoplasias malignas de outras localizações, de localização mal definida, secundárias e de localização não especificada e neoplasia maligna do cólon.

\section{Discussão}

Nas últimas décadas tem-se observado mudança no perfil demográfico e epidemiológico da população, concomitante ao aumento da expectativa de vida da população e de doenças crônicas. Sabe-se ainda que a carga das neoplasias se apresenta elevada no Brasil e medidas públicas de caráter populacional devem ser priorizadas para o efetivo controle da morbidade e da mortalidade por este agravo. Em países desenvolvidos, um em cada cinco óbitos é decorrente de neoplasias, todavia, em países em desenvolvimento esta proporção equivale a 10\% 10 .

Relacionado a principal causa de mortalidade por neoplasias foi encontrado Rstante de neoplasias malignas - descrita a partir de códigos do CID-10 que em alguns ersam sobre localizações mal/ não definidas/ especificadas. Matos et al. ${ }^{15}$, destaca que evem ser avaliadas as altas taxas de mortalidade uma vez que podem estar 
relacionadas pelo diagnóstico tardio e pelo atraso na implantação do tratamento adequado.

Quanto a neoplasia maligna da mama, segunda causa de mortes por neoplasia no quinquênio, autores destacam que existem importantes diferenças no país, seja na distribuição da oferta e utilização dos procedimentos de detecção precoce do câncer de mama, seja na cobertura e no desequilíbrio entre o que é produzido e a necessidade social estimada, de modo que observa-se o descompasso entre o estímulo à realização da mamografia e a estrutura correspondente para investigação diagnóstica, evidenciando um grande estrangulamento da capacidade diagnóstica verificada na rede de serviços do SUS16.

Destaca-se que as desigualdades regionais na mortalidade por câncer atribuíveis às variações nos fatores socioeconômicos e na disponibilidade e acessibilidade dos serviços de detecção e tratamento precoce ${ }^{17}$. Relacionado às diferenças nas taxas de incidência e mortalidade por câncer de mama nos países e suas regiões, estas justificam-se não só por fatores biológicos, ambientais, comportamentais e sociais, mas também pelas estratégias governamentais adotadas para prevenção, detecção e diagnóstico precoces ${ }^{18}$.

Corroborando com tais achados, autores destacam que as razões entre mamografia, PAAF e PAG e a população de mulheres, de modo geral, aumentaram, enquanto as razões de biópsia diminuíram em várias regiões, exceto no Sul e no Centro-Oeste, fato que indica que, na maior parte do país, a rede pública não supriu as necessidades de biópsia, procedimento essencial para confirmação de neoplasias malignas da mama, o que pode estar relacionado à baixa disponibilidade de recursos humanos para realização desse procedimento ${ }^{16}$.

Matos et al. ${ }^{15}$ informa que o aumento no número de internações e óbitos na faixa etária justifica-se parcialmente pelas características individuais como a idade, que se constitui um dos mais importantes fatores de risco para o desenvolvimento e rognóstico da neoplasia maligna da mama. Destarte ainda que o câncer de mama é antes dos 30 anos de idade, tem aumento da sua incidência até os 50 anos, todavia, 
tem sido observada em todo o mundo, uma elevação da incidência dessa neoplasia, inclusive em faixas etárias mais jovens.

A idade é uma importante fonte de variação nas taxas por causa do aumento do risco de morrer com o processo biológico do envelhecimento ${ }^{19} \mathrm{e}$, ainda, contribui para o desenvolvimento de vários tipos de tumores ${ }^{20}$. Nesta pesquisa observa resultados corroborados por tais achados, uma vez que se verificou maior mortalidade a partir do grupo etário de 30 anos ou mais. Portanto, sinaliza-se a necessidade de direcionar esforços para suprir as lacunas presentes na linha de cuidado do câncer de mama, e a importância de análises locais similares para apurar o diagnóstico, objetivando a melhor estruturação da rede de atenção à saúde.

O estudo traz que as mais altas taxas de mortalidades estavam na faixa etária de 50 a 59 anos, de modo que considerar a faixa-etária é uma estratégia importante para que se atinjam os objetivos pretendidos com as ações de controle da doença e de detecção precoce e para a programação dos cuidados, ainda que nem todas as neoplasias relacionadas entre as cinco mais recorrentes por faixa etária sejam passíveis de ações de detecção precoce, como, por exemplo, encéfalo e pulmão ${ }^{21}$.

Quanto à morbidade hospitalar destaca-se a necessidade da atenção integral ao usuário, com vistas a robustez da Atenção Primária à Saúde ocorrer concomitantemente a um sistema de saúde fortalecido com um rearranjo políticoorganizativo-técnico-assistencial de caráter contínuo e sistêmico. Ressalta-se portanto a necessidade de fortalecer e expandir a rede de tratamento do câncer no SUS para todas as regiões do Brasil.

Quanto a internação hospitalar por neoplasias, Góis e Veras²2 destacam que no período de 10 anos por estes autores avaliados, houve significativo aumento de internações hospitalares o que demonstra ser necessário pesquisar e sinalizar, tanto para o governo quanto para a sociedade e recomenda, por medida de precaução, informar aos serviços de saúde as neoplasia mais prevalentes, no sentido de alertar ao rofissional de saúde para estar atento à detecção da forma mais precoce possível, itando o aumento desses números. 
As medidas de cobertura são importantes para o monitoramento global porque respondem mais rapidamente do que as medidas de impacto às intervenções de políticas e programas ${ }^{23}$. Os indicadores de saúde apresentam-se como cruciais para a área da saúde, visto que saber sobre o número de óbitos, suas causas e condições, bem como de outros eventos, fornece informações essenciais para o estabelecimento de metas e avaliação dos planos sociais e econômicos após sua análise e interpretação ${ }^{24}$.

\section{Conclusões}

Destarte, foi observado com os maiores números de mortalidade por neoplasia está relacionado a Neoplasia maligna de mama, outrora, as morbidades estejam associadas a Leiomioma do útero. Sendo assim a análise de indicadores da saúde por neoplasias demonstra a tendencia crescente no quinquênio da morbidade hospitalar e mortalidade. De modo que se destacam que sejam alvo de maiores pesquisas e atenções.

Portando, se faz necessário, urgentemente, a ampliação das medidas de planejamento e avaliação propostas, avaliando a assistência oferecida, caracterizando a população assistida, conhecendo a qualidade das ações de saúde, e observando as fragilidades para a prestação de uma assistência de qualidade aos usuários e, principalmente, reestruturar e fortalecer as políticas e os programas de saúde, com vistas a direcionar as intervenções para as reais necessidades e obter melhores indicadores.

\section{Referências}

1. Brasil. Ministério da Saúde. Plano de ações estratégicas para o enfrentamento das doenças crônicas não transmissíveis (DCNT) no Brasil, 2011-2022. Brasília: Ministério da Saúde; 2011.

2. World Health Organization. Global status report on noncommunicable diseases 2010. Geneva: WHO; 2011.

3. World Health Organization. Preventing chronic diseases: a vital investment.; Geneva: WHO 2005. 
4. World Health Organization. International Agency for Research on Cancer. World Cancer Report 2008. Lyon: IARC Press; 2008.

5. Instituto Nacional de Câncer José Alencar Gomes da Silva. ABC do Câncer: Abordagens Básicas para o Controle do Câncer. Rio de Janeiro: INCA; 2019.

6. Bray F, Ferlay J, Soerjomataram I, Siegel RL, Torre LA, Jemal A. Global cancer statistics 2018: GLOBOCAN estimates of incidence and mortality worldwide for 36 cancers in 185 countries. CA Cancer J Clin. 2018;68(6):394-424.

7. Rezende LFM, Lee DH, Louzada MLC, Song M, Giovannucci E, Eluf-Neto J. Proportion of cancer cases and deaths attributable to lifestyle risk factors in Brazil. Cancer Epidemiol. 2019;59:148-57.

8. Ferlay J, Soerjmataram I, Dikshit R, Eser S, Mathers C, Rebelo M, et al. Cancer incidence and mortality worldwide: sources, methods and major patterns in GLOBOCAN 2012. Int J Cancer. 2015;136(5):359-86.

9. Pilleron S, Sarfati D, Janssen-Heijnen M, Vignat J, Ferlay J, Bray F, et al. Global cancer incidence in older adults, 2012 and 2035: a population-based study. Int J Cancer. 2019;144(1):49-58.

10. Boing AF, Vargas SAL, Boing AC. A carga das neoplasias no Brasil: mortalidade e morbidade hospitalar entre 2002-2004. Rev. Assoc. Med. Bras. [Internet]. 2007 Aug; 3(4):317-322.

11. Bishehsari F, Mahdavinia M, Vacca M, Malekzadeh R, Mariani-Costantini R. Epidemiological transition of colorectal cancer in developing countries: Environmental factors, molecular pathways, and opportunities for prevention. World J Gastroenterol. 2014; 20(20): 6055-72.

12. Sierra MS, Forman D. Burden of colorectal cancer in Central and South America. Cancer Epidemiol. 2016; 44: S74-81.

13. Instituto Brasileiro de Geografia e Estatística. Pesquisa Nacional de Saúde 2013: percepção do estado de saúde, estilos de vida e doenças crônicas: Brasil, grandes regiões e unidades da federação. Rio de Janeiro: Instituto Brasileiro de Geografia e Estatística; 2014.

14. Brasil. Resolução $n^{0}$ 510, de 07 de abril de 2016. Dispõe sobre as normas aplicáveis a pesquisas em Ciências Humanas e Sociais. Diário Oficial da República Federativa do Brasil, Brasília, DF, 24 maio 2016.

15. Matos BES, Pereira NAM, Rocha FC, Brasil CA, Cardoso ACC, Palmeira CS. Caracterização de mulheres hospitalizadas por neoplasia maligna da mama na Bahia, Brasil, 2012-2016. Rev Enferm Contemp. 2020;9(1):50-57. 
16. Tomazelli JG, Silva GA. Rastreamento do câncer de mama no Brasil: uma avaliação da oferta e utilização da rede assistencial do Sistema Único de Saúde no período 2010-2012. Epidemiol. Serv. Saúde. 2017; 26( 4 ): 713-724.

17. Azevedo e Silva G, Bustamante-Teixeira MT, Aquino EM, Tomazelli JG, DosSantos-Silva I. Acesso à detecção precoce do câncer de mama no Sistema Único de Saúde: uma análise a partir dos dados do Sistema de Informações em Saúde. Cad Saude Publica. 2014;30(7):1537-50.

18. Figueiredo FWS, Adami F. Effects of the high-inequality of income on the breast cancer mortality in Brazil. Sci Rep. 2019;9(1):8-13.

19. Clayton D, Schifflers E. Models for temporal variation in cancer rates. I: ageperiod and age-cohort models. Stat Med 1987; 6:449-67

20. Richardson B. Impact of aging on DNA methylation. Ageing Res Rev 2003; 2:245-61.

21. World Health Organization. Cancer control: knowledge into action: WHO guide for effective programmes; module 3. Geneva: World Health Organization; 2007.

22. Góis ALB, Veras RP. Informações sobre a morbidade hospitalar em idosos nas internações do Sistema Único de Saúde do Brasil. Ciênc. saúde coletiva. 2010 Set; 15(6): 2859-2869.

23. Ruducha J et al. How ethiopia achieved millennium development goal 4 through multisectoral interventions: a countdown to 2015 case study. Lancet Glob Health. 2017; 5(11):e1142-e1151.

24. Martins PCR, Pontes ERJC, Higa LT. Convergência entre as Taxas de Mortalidade Infantil e os Índices de Desenvolvimento Humano no Brasil no período de 2000 a 2010. Interações (Campo Grande). 2018; 19(2):291-303. 